\title{
New Approaches to Liquid Interfaces through Changes in the Refractive Index and Nonlinear Susceptibility Utilizing Ultrashort Laser Pulses
}

\author{
Hiroharu YUI, $* * * \dagger$ Yasushi HIRose, $* *$ and Tsuguo SAWADA $* * * *$ \\ *CREST, Japan Science and Technology Agency (JST), Nanoarchitectonics Research Center (NARC), \\ National Institute of Advanced Industrial Science and Technology (AIST), Tsukuba Central 4, 1-1-1 \\ Higashi, Tsukuba, Ibaraki 305-8562, Japan \\ **Department of Advanced Materials Science, Graduate School of Frontier Sciences, The University of Tokyo, \\ 5-1-5-603, Kashiwanoha, Kashiwa, Chiba 277-8561, Japan
}

\begin{abstract}
Molecules in inhomogeneous liquid environments, such as air/liquid, liquid/liquid, solid/liquid interfaces interact with each other specifically, and sometimes form characteristic structures and emerge unique properties. Here, we introduce two newly developed spectroscopic techniques, the total-internal-reflection ultrafast transient lens method (TIR-UTL) and second harmonic generation-coherent vibrational spectroscopy (SHG-CVS), to investigate the characteristic behaviors of molecules in such inhomogeneous environments. TIR-UTL probes the refractive-index change with sub-picosecond resolution and provides information on ultrafast changes in the population, density, and thermal properties, such as temperature increase and energy transfer from the solute molecules to the surrounding solvent molecules. On the other hand, SHG-CVS probes nonlinear susceptibility changes at the interfacial areas, and is expected to provide spectroscopic information on the low-frequency vibrational modes that reflect the corrective motion of the molecules in such an inhomogeneous environment. These new approaches are based on pump-probe techniques utilizing (ultra) short laser pulses. They are expected to provide further information on inhomogeneous environments from the viewpoints of solutesolvent interactions, changes in the molecular orientation, and the corrective motion of molecules at liquid interfaces.
\end{abstract}

(Received August 9, 2004; Accepted September 13, 2004)

1 Introduction

2 UTL Measurements of AOT Reversed Micelles as a Microscopic Model of an Oil/Surfactant/ Water Interface

3 Development of the Total-Internal-Reflection Ultrafast Transient Lens (TIR-UTL) Method
1493

1494

1496
4 Development of Second-Harmonic GenerationCoherent Vibrational Spectroscopy (SHG-CVS) to Measure Low-frequency Vibrational Modes 1497

5 Conclusion 1499

6 Acknowledgements 1499

7 References 1499

\section{Introduction}

Interfaces between air/liquids, two immiscible liquids, and solid/liquids are specific areas where the chemical and physical properties change significantly on a nanometer scale upon passing from the liquid phase to some other phase. ${ }^{1}$ Thus, at such an interfacial area, the characteristic behaviors and chemical reactions of molecules can be observed due to the inhomogeneous environment. Fluorescent spectroscopy has often been utilized using environment-sensitive probe molecules to clarify the local chemical and physical properties in such an inhomogeneous environment. ${ }^{2}$ We expect that it will provide further information to understand the characteristic chemical processes at interfaces to probe ultrafast dynamics, not only of

$\doteqdot$ To whom correspondence should be addressed.

E-mail: yui@molle.k.u-tokyo.ac.jp the solutes, but also of the surrounding solvent molecules in such an inhomogeneous environment with femto- and picosecond resolution. This is because not only the solute molecules, but also the interface-perturbed solvent molecules interacting with the solute molecules, should play important roles in the characteristic behavior and chemical reactions that are observed only at the interfacial areas.

We have developed an ultrafast transient lens (UTL) method to detect ultrafast changes of the refractive index through a pump-probe technique. ${ }^{3-5}$ The UTL enables us to measure the molecular dynamics of a solution as a refractive-index change of the solution. It can be used to monitor nonradiative processes sensitively with sub-picosecond time-resolution. In addition, the refractive index of a solution directly reflects the dynamics of both solute and solvent molecules. Thus, it is expected that UTL method can enable us to monitor the ultrafast dynamics of not only the solute, but also the nearby solvent molecules that are difficult to detect by time-resolved fluorescence or transient 
absorption spectroscopy. During the last decade, the UTL method has been applied to investigate the relaxation processes of energy transfer from a photoexcited solute to the surrounding solvent molecules, ${ }^{6,7}$ the ultrafast dynamics of both the solute and solvent molecules, ${ }^{8,9}$ the characteristic population change in confined systems, ${ }^{10,11}$ and so on. Therefore, we consider that the application of UTL to an inhomogeneous liquid environment provides a complementary approach to investigate the characteristic behaviors induced at liquid interfaces.

We divided the present review into three sections. The first section describes the measurement of bis(2ethylhexyl)sulfosuccinate (AOT) reversed micelles by UTL, as a microscopic oil/surfactant/water interface system to examine whether an ultrafast refractive index change can provide further information on such an inhomogeneous liquid system. Small AOT reversed micelles provide ideal models of an oil/surfactant/water interface with well-defined dimensions. The radius of an AOT reversed micelle is almost proportional to the molar ratio of water to AOT $\left(w_{0}=\left[\mathrm{H}_{2} \mathrm{O}\right] /[\mathrm{AOT}]\right)$, and can be easily controlled from several angstroms to about ten nanometers. ${ }^{12}$ Thus, they have been extensively studied as models of confined water in macromolecules, or as models of biologically relevant interfaces. ${ }^{13}$ We found that the UTL has provided further information on the corrective behaviors of such inhomogeneous environments where several kinds of molecules interact with each other. ${ }^{14,15}$

The second and third sections present the development of new instruments that we have newly developed. The second section shows the construction of a total-internal-reflection UTL (TIRUTL) instrument. The TIR-UTL instrument enables us to measure an ultrafast change in the refractive index induced at the interfacial area, where pump and probe beams are introduced from the higher refractive index phase under the total-internal-reflection configuration. ${ }^{16}$ The excitation area is limited to the adjacent area of the interface due to evanescent wave excitation. The TIR-UTL is applicable to interfaces between two transparent media with different refractive indexes.

The third section shows surface-selective coherent vibrational spectroscopy that is based on the modulation of the nonlinear susceptibility due to the coherent excitation of the lowfrequency vibrational modes. Coherent excitation can be achieved by ultrashort laser pulse irradiation that has a sufficient bandwidth for the stimulated Raman excitation mechanism. ${ }^{17}$

\section{UTL Measurements of AOT Reversed Micelles as a Microscopic Model of an Oil/Surfactant/ Water Interface}

In UTL as well as thermal lens (TL) spectroscopy, a refractive index change $(\Delta n)$ is induced by a pump beam with a spatially nonuniform intensity. Provided that a pump beam having a spatially Gaussian-type intensity is used for excitation, and that the excitation is a one-photon process, the refractive index $(n(r, t))$ in the excited region is described as

$$
n(r, t)=n_{0}+\Delta n(t)\left(1-r^{2} / w^{2}\right)
$$

where $r$ is the distance from the center of the pump beam, $n_{0}$ is the unperturbed refractive index of the solution, $w$ is the radius of the pump beam, and $\Delta n(t)$ is the photo-induced refractive index change. Here, if $\Delta n(t)>0, n(r, t)$ is higher at the center of the pump beam and the sample solution works as a "transient" convex lens. By contrast, a concave lens is formed when $\Delta n(t)$ (a)

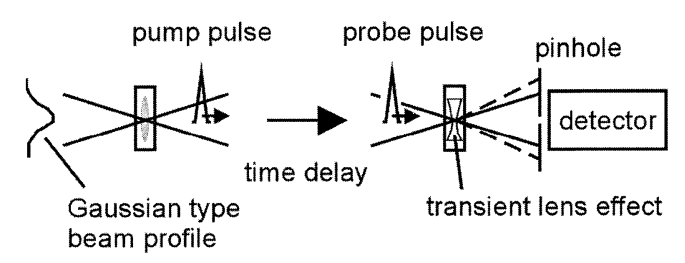

(b)

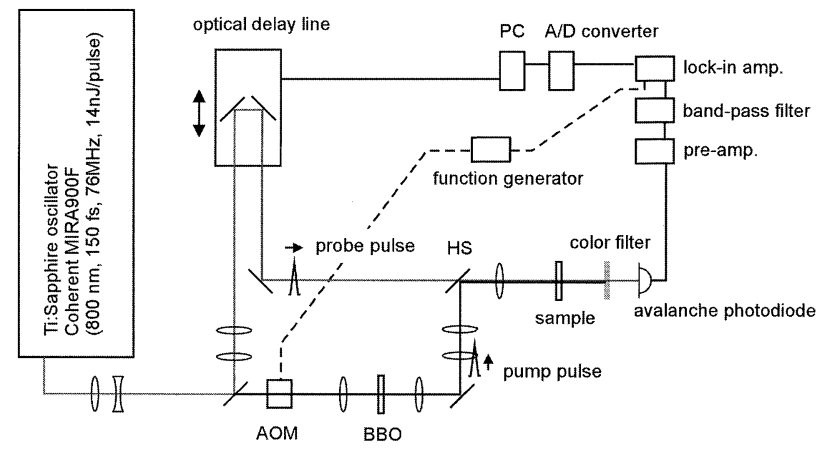

Fig. 1 (a) Schematic illustration of the principle of the UTL measurement. (b) Schematic diagram of the UTL measurement. AOM, acousto-optic modulator; BBO, BBO crystal; and HS, harmonic separator.

$<0$. These changes of the refractive index are detected as changes in the optical density of the probe beam due to their focusing and defocusing induced by the transient lens (Fig. 1(a)).

The apparatus of the UTL measurement is illustrated in Fig. 1(b). A mode-locked Ti:Sapphire laser was used as a light source (Coherent, Mira 900F; centered at $800 \mathrm{~nm}, 76 \mathrm{MHz}$ repetition, $14 \mathrm{~nJ} /$ pulse, $150 \mathrm{fs}$ FWHM). A part of the laser output was separated by a beamsplitter and used as a probe beam after passing through a computer-controlled optical delay line. The residual beam was frequency doubled by a BBO crystal to generate a pump beam. The intensity of the pump beam was repeatedly modulated with an acousto-optic modulator (AOM, frequency: $1.1 \mathrm{MHz}$ ). The pump and probe beams were set to be collinear with a harmonic separator, and focused on the sample cell. The power of the pump beam was less than $200 \mathrm{pJ} / \mathrm{pulse}$. A colored glass filter was used to cut off the pump beam at the front of the photodiode. The refractive index change was detected as a change in the probe beam intensity at the small center area of an avalanche photodiode $(\phi$ $=0.1 \mathrm{~mm}$ ). The output of the photodiode was passed through a pre-amplifier (NF, SA-430F5) and a homemade passive bandpass filter before being sent to a lock-in amplifier (EG \& G Princeton Applied Research, Model 5202) synchronized with the AOM modulation. The response function of the whole system was about 300 fs FWHM.

We prepared water/AOT/ $n$-heptane reversed micelle systems, where water existed as a nanometer-sized spherical pool surrounded by a layer of surfactant AOT molecules, whose hydrophilic head groups pointed inward (Fig. 2(a)). We measured the relaxation processes of the photoexcited Auramine $\mathrm{O}(\mathrm{AuO})$ (Fig. 2(b)) included in several sizes of the reversed micelles $(0.8,1.4,2,4$, and $8 \mathrm{~nm}$ in radius). AuO has been widely used as a viscosity probe molecule, and its relaxation process is remarkably affected by the surrounding environments. ${ }^{18}$ In addition, because $\mathrm{AuO}$ is a cationic dye and 
Table 1 Comparison of the lifetimes of LE and TICT-like states from UTL measurements and transient absorption spectra

\begin{tabular}{ccccc}
\hline $\begin{array}{c}\text { Radius of reversed } \\
\text { micelle/nm }\end{array}$ & $\tau_{1} / \mathrm{ps}^{\mathrm{a}}$ & $\tau_{2} / \mathrm{ps}^{\mathrm{a}}$ & $\tau_{\mathrm{LE}} / \mathrm{ps}^{\mathrm{b}}$ & $\tau_{\mathrm{TICT}} / \mathrm{ps}^{\mathrm{b}}$ \\
\hline Bulk water & 0.2 & - & 0.3 & 1.4 \\
8 & 2.0 & 9.6 & 1.9 & 10.4 \\
4 & 2.6 & 12.0 & 2.3 & 12.3 \\
2 & 3.0 & 15.2 & 3.7 & 15.4 \\
1.4 & 3.6 & 15.9 & 5.4 & 20 \\
0.8 & 6.0 & 26 & 8 & 30 \\
\hline
\end{tabular}

a. Estimated from UTL measurements.

b. Estimated from transient absorption measurements.

AOT is an anionic surfactant, we considered that $\mathrm{AuO}$ is preferentially located in the interfacial area due to the electrostatic interaction. Photoexcited AuO relaxes via two intermediate states, that is, the first-formed emissive locally excited (LE) state and a nonemissive twisted intramolecular charge transfer-like (TICT-like) state (Fig. 2(c)). ${ }^{19}$ The relaxation process of photoexcited $\mathrm{AuO}$ proceeds with a twisting motion of the phenyl groups. Thus, we can expect that the corrective behaviors of the water/surfactant/oil molecular system induced by the electrostatic interaction between the $\mathrm{AuO}$ in the TICT-like state and the negatively charged interface of the AOT reversed micelle can be monitored by the ultrafast change in the refractive index.

Figure 3 shows the UTL signals of photoexcited $\mathrm{AuO}$ in bulk water and AOT reversed micelles. A temporal decrease of the refractive index following the optical Kerr effect (OKE) signal was observed in the reversed micelles as well as in bulk water. However, the time evolutions of the UTL signals in reversed micelles were clearly different from that in bulk water. Table 1 gives the time constants of the UTL signals and the lifetimes of the LE and TICT-like states of AuO measured by transient absorption spectroscopy in both bulk water and in the reversed micelles. The UTL signal in bulk water was well fitted by a single-exponential function, and the time constant $\left(\tau_{1}\right)$ corresponded to the lifetime of the LE state $\left(\tau_{\mathrm{LE}}\right)$. By contrast, double-exponential functions were needed to fit the UTL signals in reversed micelles, and the time constants of the two decay components ( $\tau_{1}$ and $\tau_{2}$ ), agreed well with the lifetime of the LE and TICT state ( $\tau_{\mathrm{LE}}$ and $\left.\tau_{\mathrm{TICT}}\right)$, respectively (Table 1$)$. Thus, we assigned the $\tau_{1}$ and $\tau_{2}$ components of the UTL signal to the refractive-index change accompanying the formation of the LE and the TICT-like states, respectively.

In general, the changes in the structure and/or the electronic state of solute molecules should result in a change of the refractive index of the solution. Thus, our result indicated that the structure and/or electronic state of the TICT-like state of $\mathrm{AuO}$ in the reversed micelles were somewhat different from those in bulk water. Such changes in structure and/or the electronic state of $\mathrm{AuO}$ were also supported by the transient absorption spectra, where the absorption coefficients of the TICT-like state in the reversed micelles were about half as large as than that in bulk water (Fig. 4(a)). ${ }^{15}$ These results indicated that the interactions with the interface strongly affect the structure and/or electronic state of $\mathrm{AuO}$ adjacent to the interface.

Interestingly, a further analysis of the UTL signal revealed that another factor was also responsible for the refractive-index change due to the formation of the TICT-like state $\left(\Delta n_{\mathrm{TICT}}\right)$. In Fig. 4(b), the refractive-index change due to the formation of both the LE state $\left(\Delta n_{\mathrm{LE}}\right)$ and the $\Delta n_{\mathrm{TICT}}$ was quantitatively (a)

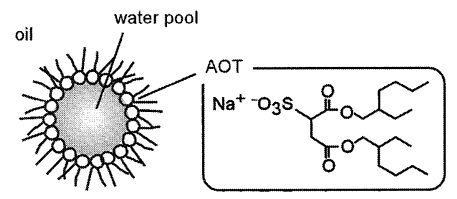

(b)

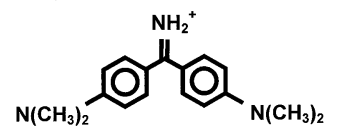

(c)

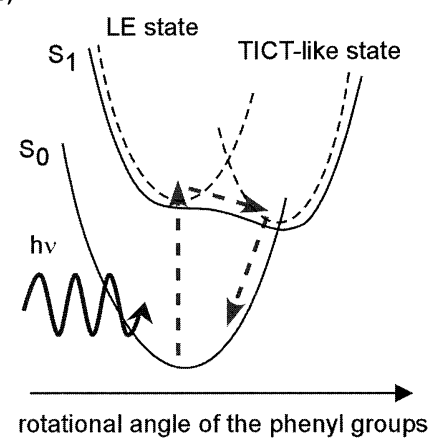

Fig. 2 (a) Schematic illustration of AOT reversed micelles and the molecular structure of AOT. (b) Molecular structure of AuO. (c) Relaxation dynamics of the AuO. Two intermediate states, LE (locally excited) and TICT (twisted intramolecular charge transfer)like states, are indicated.

evaluated by a kinetic analysis of the UTL signals. ${ }^{17,20,21}$ It was clearly shown that $\Delta n_{\mathrm{LE}}$ was independent of the micellar size, while $\Delta n_{\text {TICT }}$ increased with a decrease of the reversed micelle size. By contrast, the transient absorption spectra of the TICTlike state did not depend on the micellar size (Fig. 4(a)), indicating that the molecular structure (e.g., twisting angle of phenyl groups) and charge distribution of the TICT-like state of $\mathrm{AuO}$ molecules were independent of the micellar size. ${ }^{15}$ These results show that the size-dependent component in $\Delta n_{\text {TICT }}$ does not originate from the $\mathrm{AuO}$ molecules.

A probable candidate for the refractive-index change in $\Delta n_{\mathrm{TICT}}$ is the temperature increase of the surrounding solvent molecules. If the increase in the temperature of the surrounding water molecules is responsible for the UTL signal, decrease of the refractive index should be observed along with the decay of the TICT-like state, because $(\mathrm{d} n / \mathrm{d} T)_{\mathrm{V}}$ of water is negative. ${ }^{22}$ However, such a decrease in the refractive index, corresponding to a temperature increase, was not observed (Fig. 3(b)). Thus, the temperature increase of the surrounding water cannot be responsible for the size dependence of $\Delta n_{\text {TICT. }}$. It is worth noting that $\left|\Delta n_{\text {TICT }}\right|$ remarkably increases when the size of the reversed micelle is comparable to the size of the $\mathrm{AuO}$ molecule $(\sim 1 \mathrm{~nm})$. This result suggests that the formation of the TICT-like state induces a structural change of the reversed micelles. ${ }^{11,15,17}$ It is known that a change in the collective structure of molecules in solution (e.g. solvation structure) results in a change of the local polarizability, which is observed as a refractive-index change. ${ }^{8}$ The formation of the TICT-like intermediate should induce strong perturbations to the surrounding water and AOT molecules by the twisting motion of the phenyl groups and the intramolecular charge transfer. It is reasonable to consider that such perturbations should more strongly affect the local 

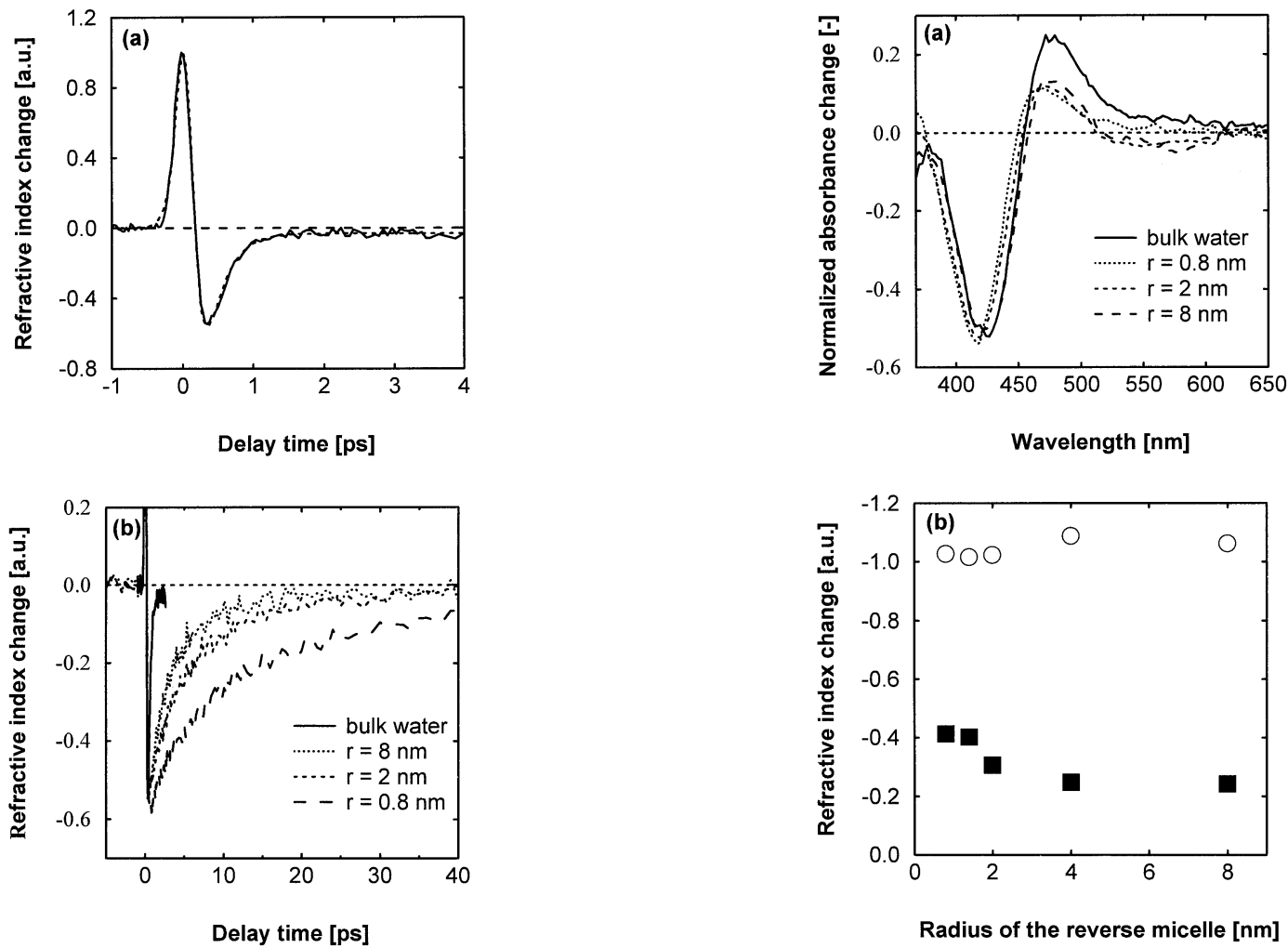

Fig. 3 (a) UTL signal of $\mathrm{AuO}$ in bulk water. (b) UTL signals of $\mathrm{AuO}$ in AOT reversed micelles with various radiuses $(0.8 \mathrm{~nm}, 2 \mathrm{~nm}$, and $8 \mathrm{~nm}$ ).

structure of the reversed micelles, especially with smaller diameters. Although further experiments should be carried out to confirm the assignment, we observed these signals, which were not detected by transient absorption measurements. The change in the refractive index will provide further information on the local environment, including not only solute molecules, but also the surrounding solvent molecules. This is a merit to investigate an inhomogeneous environment, such as air/liquid, liquid/liquid, and solid/liquid interfaces, where interfaceperturbed solvent molecules play important roles in interfacespecific reactions and behaviors.

\section{Development of the Total-Internal-Reflection Ultrafast Transient Lens (TIR-UTL) Method}

Next, we developed a total-internal-reflection ultrafast transient lens (TIR-UTL) method to apply the UTL technique to interfaces between two immiscible media. ${ }^{16}$ In the TIR-UTL measurement, pump and probe beams are coaxially introduced onto the interfaces by a total-internal-reflection (TIR) configuration. Irradiation by the TIR configuration enables us to limit the excitation area adjacent to the interfaces, and thus to drastically reduce the contribution from molecules in the bulk phase. We measured ultrafast dynamics of auramine-O (AuO) molecules at a water/silica interface by the TIR-UTL method, and compared the result with that in bulk water. We expected that we could detect the characteristic dynamics of $\mathrm{AuO}$ molecules that adsorbed on a silica surface, with their twisting motions being strongly hindered.

The TIR-UTL method utilizes a pump-probe technique to achieve a subpicosecond time resolution. Figure 5(a) shows the

Fig. 4 (a) Transient absorption spectra of $\mathrm{AuO}$ in bulk water and in reversed micelles with various radiuses $(0.8 \mathrm{~nm}, 2 \mathrm{~nm}$, and $8 \mathrm{~nm})$. To exclude the overlap of the gain band of the LE state on the transient absorption band of the TICT-like state, all spectra were normalized at the delay time when the bleaching band recovered to $50 \%$ (the gain band completely decayed at that time). (b) Refractive index change of the reversed micelles accompanying the formation of the LE state (open circle) and the TICT-like state (filled square). $\Delta n_{\mathrm{LE}}$ and $\Delta n_{\mathrm{TICT}}$ were evaluated by a kinetic analysis of the following two-step relaxation that was indicated in our previous study. ${ }^{15}$

principle of the TIR-UTL. When a pump beam is incident on a hemispherical prism (fused silica; $\phi=15 \mathrm{~mm}$ ), an evanescent field is generated near the interface. The penetration depth of the evanescent field to medium on the sample side $(d p)$ is defined by

$$
d p=\frac{\lambda}{2 n_{1} \pi \sqrt{\sin ^{2} \theta-n_{12}{ }^{2}}} .
$$

Here, $n_{1}$ is the refractive index of the medium on the prism side. $n_{2}$ is the refractive index of the medium on the sample side. $n_{12}$ is $n_{2} / n_{1}$, the relative refractive index. $\theta$ is the incident angle. $\lambda$ is the wavelength of the incident light. The evanescent field of the pump beam generates a spatial distribution of the ultrafast refractive index change near the interface. The ultrafast refractive index change, which originates from photochemical phenomena, was detected by the intensity change of the center area of the probe beam. The change in the intensity was detected by an avalanche photodiode. The theoretical principle of the TIR-UTL technique is described elsewhere. ${ }^{16}$

The light source and the optical arrangement for the TIR-UTL set-up were the same as those for the UTL measurement, except for the arrangement of the laser beams and the samples. We fabricated the original sample-prism unit (Fig. 5(b)) and adjusted the incident angle and the focal point of the pump and probe beams on the interface by using an $x y z \theta$ stage. The 
(a)

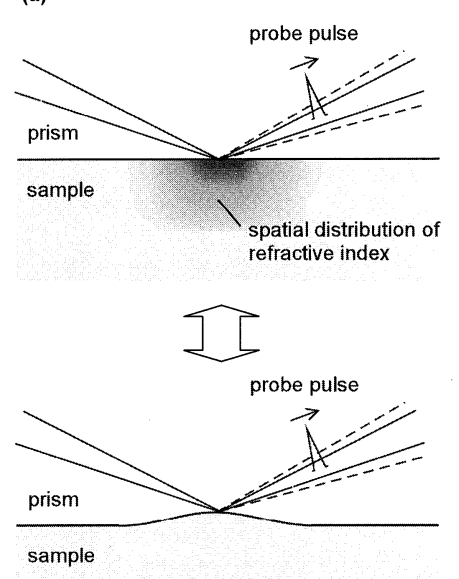

(b)

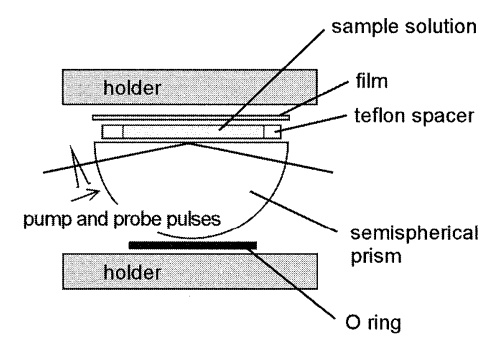

Fig. 5 (a) Schematic illustration of the beam deflection by a total reflection at the interface with a spatially nonuniform refractive index. The spatially nonuniform refractive index change is equivalent to the spatial deformation of the sample media. (b) Schematic illustration of the sample cell unit and the light incident condition for the TIR-UTL measurement.

incident angle of the beams was set at $\theta=86^{\circ}$, and $d p$ was calculated as $100 \mathrm{~nm}$ from Eq. (2). The polarizations of the beams were set to be parallel. Since $\mathrm{AuO}$ is a cationic dye, $\mathrm{AuO}$ is adsorbed on a silica surface, which is negatively charged by the ionization of silanol groups in water. The concentration of $\mathrm{AuO}$ was $0.40 \mathrm{mM}$ and $1.0 \mathrm{mM}$ for UTL and TIR-UTL measurements, respectively.

We then compared the UTL and TIR-UTL signals of AuO aqueous solution to confirm whether the latter come from the interfacial molecular dynamics. The UTL and the TIR-UTL signals of the $\mathrm{AuO}$ aqueous solution are shown in Fig. 6. Regarding both signals, we observed an instantaneous increase and subsequent decrease of the refractive index. These signals were attributed to the OKE of the prism and water, and the relaxation dynamics of $\mathrm{AuO}$, respectively. As discussed above, the UTL signal of AuO in bulk water was well fitted by a single exponential function with a time constant of $\tau_{1}<400 \mathrm{fs}$. In contrast, the TIR-UTL signal was fitted by the sum of three exponential functions with time constants of $\tau_{1}<400 \mathrm{fs}, \tau_{2}=4.5$ ps, and $\tau_{3}=68 \mathrm{ps}$.

The time constant of $\tau_{1}(<400 \mathrm{fs})$ was observed in both measurements. Thus, it was attributed to the relaxation process of $\mathrm{AuO}$ in bulk solution: internal conversion from the LE state to the TICT-like state. This is because the TIR-UTL signal includes a signal originating from the relaxation process of $\mathrm{AuO}$ in the bulk region, since the detection depth of our TIR-UTL system $(\sim 100 \mathrm{~nm}$ from the interface) is larger than the adsorption layer on a molecular scale. On the other hand, the two slower time constants ( $\tau_{2}$ and $\tau_{3}$ ) were observed only in the

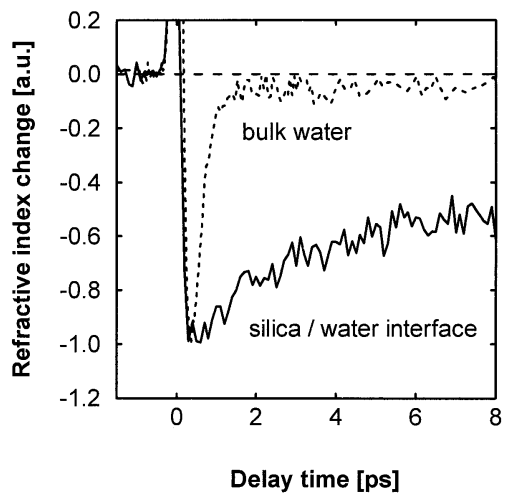

Fig. 6 Comparison of the UTL signal of AuO in bulk water and the TIR-UTL signal of AuO at a silica/water interface.

TIR-UTL signal. Thus, these slower time constants were attributed to the dynamics of $\mathrm{AuO}$ perturbed at the interface, which indicated that the characteristic molecular dynamics at the solid/liquid interface was successfully observed through the change in the refractive index by the TIR-UTL technique.

As mentioned above, since the silica surface is negatively charged in water by the ionization of silanol groups, the cationic dye, AuO, adsorbs on the silica surface by an electrostatic interaction. We then considered that the adsorption on the silica surface strongly hindered the twisting motion of the phenyl groups of $\mathrm{AuO}$, as confined in AOT reversed micelles. We interpreted the slower two components of the TIR-UTL signal according to the following two models: (1) The internal conversion process from the LE to the TICT-like state of AuO adsorbing on the interface corresponds to $\tau_{2}$ and the relaxation process from the TICT-like state to the ground state corresponds to $\tau_{3}$. Such a refractive-index change was also observed in highviscosity solvents ${ }^{4}$ and in AOT reversed micelles. ${ }^{14,15}$ (2) There are two kinds of $\mathrm{AuO}$ that interact differently with the interface. The internal conversion from the LE to the TICT-like state of each kind of $\mathrm{AuO}$ was responsible for two decay components. In both cases, the rate of the relaxation became 10 to 100-times slower, which indicated that the interaction between $\mathrm{AuO}$ and the interface strongly hindered the molecular motion of the AuO. These results indicated that TIR-UTL provides us a new method to investigate the molecular interactions and dynamics at a liquid interface.

\section{Development of Second-Harmonic Generation- Coherent Vibrational Spectroscopy (SHG- CVS) to Measure Low-frequency Vibrational Modes}

Finally, we introduce the development of a new instrument that can monitor the low-requency vibrational modes of molecules at liquid interfaces. The low-frequency vibrational region is considered to sensitively reflect the corrective motion and structures of molecular systems..$^{23-27}$ Thus, surface-selective low-frequency vibrational spectroscopy will provide qualitatively new information on the molecular dynamics and structures at an inhomogeneous liquid interface, where specific solute-solvent interactions play important roles in surfacespecific chemical processes. Representative interface-selective vibrational spectroscopy is sum frequency generation (SFG). ${ }^{28,29}$ However, there is no appropriate light source to obtain a lowfrequency vibrational spectrum by SFG. Thus, we combined 
(a)

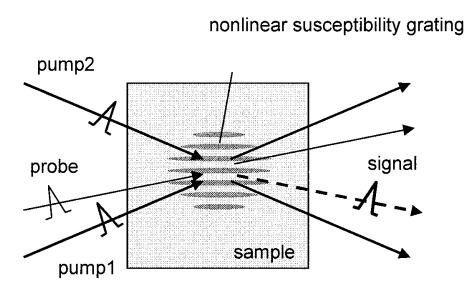

(b)

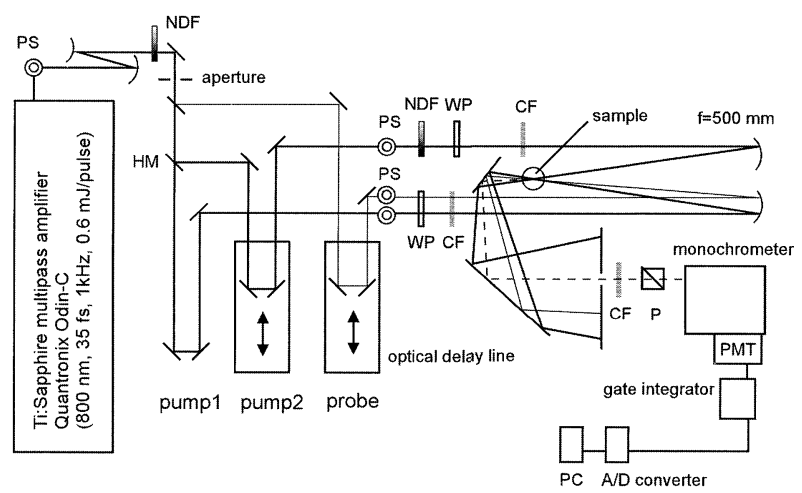

Fig. 7 (a) Schematic illustration of the principle of the SHG-CVS measurement. (b) Schematic diagrams of the experimental set-up for SHG-CVS measurement. PS, periscope; NDF, neutral density filter; $\mathrm{HM}$, half mirror; WP, $1 / 2$ waveplate; $\mathrm{CF}$, color filter; $\mathrm{P}$, polarizer; and PMT, photomultiplier.

the second-harmonic generation (SHG) technique ${ }^{29}$ and coherent vibrational spectroscopy ${ }^{30}$ to realize surface-selective low-frequency vibrational spectroscopy.

An ultrashort pulse with a few tens of femtoseconds in duration has an energy bandwidth of over a few hundred $\mathrm{cm}^{-1}$. Thus, we can induce coherent excitation of low-frequency Raman-active modes within the bandwidth of the excitation beam by stimulated Raman processes by utilizing such an ultrashort pulse. The macroscopic modulation of the linear and nonlinear susceptibility with the eigenfrequency can be induced by the coherent vibration. When one monitors the modulation of the nonlinear susceptibility $\left(\chi^{(2)}\right)$ by a time-resolved SHG measurement, one can selectively observe the low-frequency vibrational modes of molecules at the interfaces.

Figure 7(a) shows the optical-beam configuration at a liquid surface. The liquid surface was irradiated by two pump pulses with a transient grating (TG) configuration. The TG configuration remarkably improved the signal-to-background $(S / B)$ ratio of the present study. The spatial modulation of $\chi^{(2)}$ was periodically induced, like grating formed by an optical interference of the two pump pulses. We then monitored the coherent-vibration-induced modulation of the second-harmonic (SH) signals in the time domain by a delay-controlled probe beam. Since the modulation of $\chi^{(2)}$ is induced in grating-like pattern, we can separate the SH signal modulated in the time domain spatially from non-modulated $\mathrm{SH}$ signals of the probe beams. Figure 7(b) shows the experimental set-up of the SHGCVS measurement. A multipass-amplified Ti:Sapphire laser (wavelength, $800 \mathrm{~nm}$; pulse duration, $30 \mathrm{fs}$; energy, 0.6 $\mathrm{mJ} /$ pulse; repetition ratio, $1 \mathrm{kHz}$ ) was used as the light source. The optical configuration was principally the same as that for the time-resolved SHG measurement. To excite the sample with the TG configuration, we divided the pump beam into two (a)

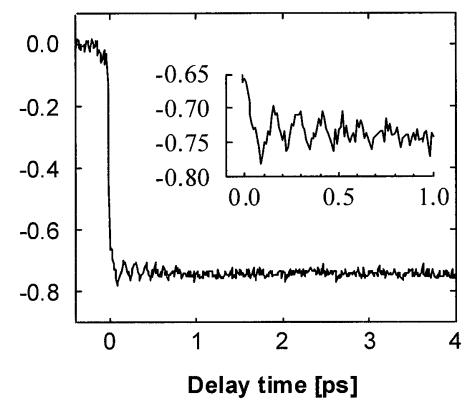

(b)

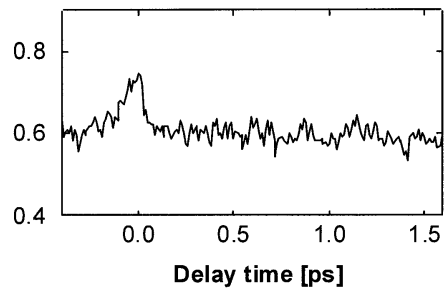

(c)

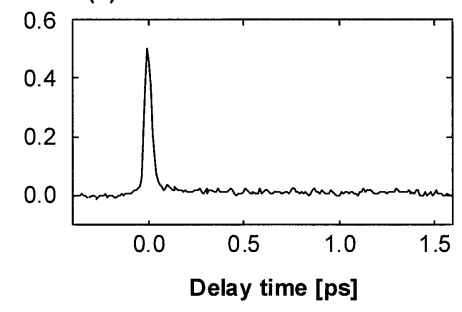

Fig. 8 (a) SHG-CVS signal of the surface phonon mode of GaAs (100). The inset is a magnified image of the observed temporal modulation of the SH signal due to the coherent vibration of the surface phonon mode. (b) SHG-CVS signal from coumarin 314 at an air/water interface. The signal duration was $130 \mathrm{fs}$ (FWHM). (c) Response function of the SHG-CVS signal measured at the surface of gold film. The signal duration was $50 \mathrm{fs}$ (FWHM).

beams, and crossed them at the sample interface. The probe beam was introduced with the phase-matching condition. The generated SH signal was detected by a photomultiplier and a gate integrator. The measurable bandwidth was estimated to be above $300 \mathrm{~cm}^{-1}$.

At first, we measured the low-frequency (phonon) mode of the surface of GaAs (100) as a test sample (Fig. 8(a)). The damped oscillation of the SH signal just after excitation was observed. The frequency of the oscillation was $275 \mathrm{~cm}^{-1}$, which can be assigned to the surface-localized phonon mode of GaAs. ${ }^{31}$ We then measured coumarin 314 at the air/water interface (Fig. 8(b)). We observed a sharp signal at delay time 0 . The duration of the signal ( $130 \mathrm{fs}, \mathrm{FWHM})$ was longer than instrumental function (50 fs, FWHM, measured at gold surface; Fig. 8(c)). Although the attribution of the signal is not yet clear, we suggested that the signal could be attributed to the inertial response and change in the orientation of the coumarin 314 due to the electrostatic fields of the pumping beam at the interface. We demonstrated that the SHG-CVS enables us to measure low-frequency vibrational spectra with surface selectivity. A further increase of the $S / N$ ratio of the SHG-CVS instrument will provide information on low-frequency vibrational modes that sensitively reflect corrective motions of molecules at liquid/liquid interfaces. 


\section{Conclusion}

We introduced new approaches to investigate the molecular dynamics at liquid interfaces through the changes in the refractive index and the nonlinear susceptibility. These approaches provide new information on a wider region than those that measure the change of probe molecules localized at interfaces. UTL measurements on $\mathrm{AuO}$ in $\mathrm{AOT}$ reversed micelles indicated that the corrective motion was induced by the electrostatic interaction between cationic solute molecules in the water pool of the AOT reversed micelles and negatively charged AOT molecules at the interface. The TIR-UTL instrument was newly developed, and was used to monitor the molecular dynamics at a solid/liquid interface. To obtain further information on the corrective motion of molecules at liquid interfaces, we also developed SHG-CVS spectroscopy utilizing ultrashort pulses. We measured the phonon-mode of the surface of GaAs and the ultrafast response of coumarin 314 at the air/water interface. Though the SHG-CVS instrument needs further improvement to apply to liquid/liquid interfaces, the spectra of low-frequency modes based on the corrective motion of the molecules will provide further information on the inhomogeneous liquid environment, where several kinds of solute and solvent molecules interact with each other and form specific structures and emerge characteristic properties.

\section{Acknowledgements}

Mr. Tsuyoshi Sugimoto is acknowledged as a co-worker in this research. These studies were supported by Japan Science and Technology Agency (JST) and Grant-in-Aids for Scientific Research on Priority Areas (No. 13129203), (B)(1)(No. 14340189), and (B)(2)(No. 14350442) by the Ministry of Education, Science, Sports, and Culture in Japan.

\section{References}

1. See, for example, A. G. Volkov, D. W. Deamer, D. L. Tanelian, and V. S. Martin, "Liquid Interface in Chemistry and Biology", 1998, John Wiley and Sons, Inc., New York.

2. See, for example, W. H. Steel and R. A. Walker, Nature, $\mathbf{2 0 0 3}, 424,296$

3. K. Ito, M. Mutoh, A. Harata, and T. Sawada, Chem. Phys. Lett., 1997, 275, 349.

4. G. Furui, K. Ito, I. Tsuyumoto, A. Harata, and T. Sawada, J. Phys. Chem. A, 1999, 103, 7575 .

5. K. Ito, I. Tsuyumoto, A. Harata, and T. Sawada, Chem.
Phys. Lett., 2000, 318, 1.

6. M. Terazima, Chem. Phys., 1994, 230, 87.

7. M. Terazima, J. Chem. Phys., 1996, 105, 6587.

8. Y. Hirose, H. Yui, M. Fujinami, and T. Sawada, Chem. Phys. Lett., 2001, 341, 29.

9. Y. Hirose, H. Yui, M. Fujinami, and T. Sawada, Anal. Sci., 2001, 17, i107.

10. M. Takei, H. Yui, Y. Hirose, and T. Sawada, J. Phys. Chem. A, 2001, 105, 11395.

11. H. Yui, M. Takei, Y. Hirose, and T. Sawada, Rev. Sci. Instrum., 2003, 74, 907.

12. M. Hirai, R. Kawai-Hirai, M. Sanada, H. Iwase, and S. Mitsuya, J. Phys. Chem. B, 1999, 103, 9658.

13. C. H. Cho, M. Chung, J. Lee, T. Nguyen, S. Singh, M. Vedamuthu, S. Yao, J.-B. Zhu, and G. W. Robinson, J. Phys. Chem., 1995, 99, 7806.

14. Y. Hirose, H. Yui, M. Fujinami, and T. Sawada, Rev. Sci. Instrum., 2003, 74, 898.

15. Y. Hirose, H. Yui, and T. Sawada, J. Phys. Chem. B, 2004, 108, 9070.

16. T. Sugimoto, Y. Hirose, H. Yui, and T. Sawada, Anal. Chem., 2004, 76, 3794.

17. (a) Y. Hirose, Doctoral Thesis, 2003, University of Tokyo; (b) in preparation.

18. M. Hasegawa, T. Sugimura, Y. Shindo, and A. Kitahara, Colloid Surf. A, 1996, 109, 305.

19. M. Glasbeek, H. Zhang, and M. J. van der Meer, J. Mol. Liq., 2000, 86, 123.

20. Y. Hirose, H. Yui, and T. Sawada, Chem. Rec., accepted.

21. We assumed first-order reaction at each relaxation process from the LE and the TICT-like state and the reaction proceed sequentially.

22. M. Terazima, J. Chem. Phys., 1996, 104, 4988.

23. R. I. Hollingsworth, J. Phys. Chem., 1995, 99, 3406.

24. Y. Amo and Y. Tominaga, Chem. Phys. Lett., 2000, 320, 703.

25. K. Egashira and N. Nishi, J. Phys. Chem. B, 1998, 102, 4054.

26. D. M. Carey and G. M. Korenowski, J. Chem. Phys., 1998, 108, 2669.

27. Y. Amo and Y. Tominaga, Chem. Phys. Lett., 2000, 320, 703.

28. D. E. Gragson and G. L. Richmond, J. Phys. Chem. B, 1998, 102, 3847 and references therein.

29. K. B. Eisenthal, Chem. Rev., 1996, 96, 1343 and references therein.

30. See, for example, L. Dhar, J. A. Rogers, and K. A. Nelson, Chem. Rev., 1994, 94, 157.

31. Y. M. Chang, L. Xu, and H. W. K. Tom, Chem. Phys., 2000, 251, 283. 